\title{
Correlation of virtual touch tissue quantification and liver biopsy in a rat liver fibrosis model
}

\author{
ZHIWEN HU ${ }^{1,2}$, JIALUN LUO ${ }^{3}$, HONGQIN WEI $^{2}$, WENCAI OU ${ }^{2}$, SHUYI XIAO ${ }^{2}$, MAN GAN $^{2}$, SUIHONG MA $^{2}$, \\ JINGGUANG HE ${ }^{2}$, DAIHONG WU ${ }^{2}$, GUIYING FENG ${ }^{2}$, JINGLU WEI ${ }^{2}$ and JIANHUA LIU ${ }^{2}$ \\ ${ }^{1}$ Medical School, Jinan University, Guangzhou, Guangdong 510632; ${ }^{2}$ Department of Medical Ultrasound, \\ Guangzhou First People's Hospital, Guangzhou Medical University; ${ }^{3}$ College of Health Sciences, \\ Guangzhou Medical University, Guangzhou, Guangdong 510180, P.R. China
}

Received August 18, 2013; Accepted November 19, 2014

DOI: $10.3892 / \mathrm{mmr} .2015 .3209$

\begin{abstract}
Liver fibrosis assessment is very important to the treatment of chronic liver disease. In the present study, Virtual Touch Tissue Quantification (VTQ) and eSie Touch ${ }^{\text {TM }}$ elasticity imaging techniques were used to examine the rat liver fibrosis model. Rat liver fibrosis was induced with thioacetamide and the degree of liver fibrosis was determined using pathological diagnosis as a gold standard. The right lobe of the liver was also examined with the VTQ and eSie Touch ${ }^{\mathrm{TM}}$ techniques. The VTQ and serological results were correlated and analyzed. The results were compared with those obtained from liver biopsies to investigate the accuracy and diagnostic value of eSie Touch ${ }^{\mathrm{TM}}$ and VTQ on the classification of liver fibrosis in rats. A total of 30 successful modeling cases were obtained, with a success rate of $86 \%$. The mean acoustic radiation force impulse (ARFI) elastography-VTQ values were $1.08,1.51,1.88$ and $2.50 \mathrm{~m} / \mathrm{sec}$ for the normal and F1/F2, F3 and F4 fibrosis groups, respectively. A significant correlation $(r=0.969)$ was identified between the ARFI measurements and the degree of fibrosis assessed by pathological examination $(\mathrm{P}<0.001)$. The histological staging results correlated with those of the eSie Touch ${ }^{\mathrm{TM}}$ elasticity imaging of the biopsy site $(r=0.913, \mathrm{P}<0.001)$. The predictive values of ARFI for various stages of fibrosis were as follows: $F \geq 1$ and 2 - cut-off $>1.250 \mathrm{~m} / \mathrm{sec}$ (when $\mathrm{Vs}>1.250 \mathrm{~m} / \mathrm{sec}$, the pathological grading was $\geq \mathrm{F} 1 / \mathrm{F} 2$ ) [Area under receiver operating characteristic $(\mathrm{AUROC})=1.00], \mathrm{F} \geq 3$ - cut-off $>1.685 \mathrm{~m} / \mathrm{sec}$ (when $\mathrm{Vs}>1.685$ $\mathrm{m} / \mathrm{sec}$, the pathological grading was $\geq \mathrm{F} 3$; AUROC $=1.00$ ) and $\mathrm{F} \geq 4$ - cut-off $>2.166 \mathrm{~m} / \mathrm{sec}$ (when $\mathrm{Vs}>2.166 \mathrm{~m} / \mathrm{sec}$, the pathological grading is cirrhosis; AUROC $=1.00$ ). In conclusion, the eSie Touch ${ }^{\mathrm{TM}}$ elasticity imaging and VTQ techniques may
\end{abstract}

Correspondence to: Dr Jianhua Liu, Department of Medical Ultrasound, Guangzhou First People's Hospital, Guangzhou Medical University, 1 Pan Fu Road, Guangzhou, Guangdong 510180, P.R. China E-mail: jianhualiucn@126.com

Key words: ultrasound, acoustic radiation force impulse, thioacetamide rat model, liver fibrosis, liver cirrhosis be successfully adopted to assess the extent of liver stiffness. These techniques are expected to replace liver biopsy.

\section{Introduction}

Liver fibrosis is a common result of chronic liver damage, which is caused by factors such as infections, toxins and autoimmune disorders, as well as cholestatic and metabolic diseases (1). At present, the global population is threatened by chronic liver diseases, with the prevalence of hepatitis B virus (HBV) infection particularly high in China. According to the World Health Organisation, $>350$ million individuals are chronically infected with HBV, which is responsible for $\sim 5.8$ million fatalities from decompensate cirrhosis or hepatocellular carcinoma annually $(2,3)$. However, accumulating evidence suggests that liver fibrosis is reversible and that recovery from cirrhosis is possible (4). Chronic liver disease involves the replacement of the hepatic parenchyma with extracellular matrix, The prognosis and management of chronic liver disease depend largely on the extent and progression of hepatic fibrosis (1). Liver biopsy (LB) remains the 'gold standard' for liver fibrosis assessment (5). However, LB has several major limitations. In removing $\sim 1 / 50,000$ total liver volume (6), LB does not completely evaluate the overall status of the liver. In addition, LB is an invasive procedure that may result in serious complications (5).

A number of studies have revealed that liver stiffness is associated with the degree of liver fibrosis. Over the past decade, non-invasive ultrasound-based diagnostic methods, including transient elastography (TE; FibroScan ${ }^{\circledR}$; Echosens, Paris, France), real-time elastography (Hitachi, Tokyo, Japan), supersonic shear wave elastography and acoustic radiation force impulse (ARFI) elastography, have been developed to replace invasive LB. TE was the first ultrasound-based elastographic method developed for liver fibrosis evaluation in patients with chronic hepatitis $\mathrm{C}$ and other etiologies of chronic hepatopathy (7). In recent years, ARFI imaging has been used as a novel ultrasound-guided elastographic method to predict liver fibrosis. In contrast to TE, ARFI permits measurements in well-defined areas of the liver tissue (8). ARFI may offer superior and more detailed diagnostic options than TE, which may only be applied in a standardised measuring position without two-dimensional (2D) image control (9). 
Virtual Touch Tissue Quantification (VTQ) is the first available technique developed to implement ARFI. The method has recently been introduced using the commercial US scanner Acuson S2000 ${ }^{\mathrm{TM}}$ (Siemens Healthcare, Erlangen, Germany) and offers the possibility of performing quantitative measurement of liver parenchyma elasticity during conventional US evaluations without requiring additional transducers or other equipment (7). In the present study, the diagnostic accuracy of VTQ (using Acuson S2000 ${ }^{\mathrm{TM}}$ ) for predicting fibrosis severity in rats with hepatitis fibrosis, compared with that of LB, was investigated.

\section{Materials and methods}

Animal model. Experiments were performed in male Sprague-Dawley rats, aged eight weeks, weighing 180-210 g, which were provided by the Guangdong Medical Laboratory Animal Centre (Guangzhou, China). Hypodermic injections of thioacetamide (TAA; Kemiou Chemical Reagent Co. Ltd., Tianjin, China) in physiological saline (Sun Yat-sen University, Guangzhou, China) were administered twice a week to induce liver fibrosis. The study was conducted in strict accordance with the recommendations in the Guide for the Care and Use of Laboratory Animals of the National Institutes of Health (Bethesda, MA, USA). The animal use procedure was reviewed and approved by the Institutional Animal Care and Use Committee of Guangzhou First People's Hospital, (Guangzhou Medical University, Guangzhou, China).

TAA saline solution (4\%) was subcutaneously injected in the abdominal wall near the hind legs. An initial dose (1 ml) of the solution was injected to induce modeling, followed by subsequent injections $(200 \mathrm{mg} / \mathrm{kg})$ twice a week. The majority of the animals reached liver fibrosis phase 2 after week 5 , then reached phases 3 and 4 on week 9 . In total, >95\% animals reached a cirrhotic state after week 12 . Liver specimens from the rats were obtained on weeks 5, 9 and 12 .

Elastography. Following successful modeling, the rats were examined using Acuson S2000 ${ }^{\mathrm{TM}}$ Color Doppler ultrasonography (Siemens Healthcare) on weeks 5, 9 and 12. A 9L4 high-frequency probe with a frequency of $9 \mathrm{MHz}$ was used. Rat hair was subsequently removed under ether anesthesia. The rats were then placed in a supine position on a mounting plate. The examined site region of interest (ROI) was the right lobe of the liver tissue, avoiding the lobe boundary and the large vessels. Semi-quantitative analysis (eSie Touch ${ }^{\mathrm{TM}}$ elasticity imaging; Siemens Healthcare) was used to assess the elastic stiffness to ensure a quality figure $(\mathrm{QF})$ value of $\geq 45$. Semi-quantitative scoring criteria for this study were produced by Yang et al (10) (Department of Ultrasonic Center, Tenth People's Hospital, Tongji University, Shanghai, China). The following five-point scale grade reference was designed by Professor Ei Ueno (University of Tsukuba, Tsukuba, Japan) (11): 1, lesion completely colored pink; 2 , lesion area mainly colored purple, with a small amount of green; 3, lesion area mainly colored green, with a small amount of yellow; 4, lesion area colored yellow, with a small amount of red; and 5, lesion area mainly colored red, with a small amount of yellow (Fig. 1). VTQ was used to measure the transverse shear wave velocity (Vs value) of the organ. The standard deviation in stiffness measurements is reduced with increasing numbers of measurements (12), therefore, the median stiffness values were recorded subsequent to seven measurements, instead of the mean values. The final sonogram and numerical data were collected and stored in a computer for statistical analysis.

Specimen collection. A total of 10 rats randomly selected from the modeling group and four rats from the control group were examined on weeks 5 and 9 using ultrasound. The rats were deeply anesthetized with phenobarbital sodium (Guangdong Bangmin Pharmaceutical Co., Ltd, Jiangmen, China), laparotomized and sacrificed. The liver mid-lobes were rapidly clipped, fixed in $10 \%$ neutral formalin-fixed solution (Guangzhou Wexis Biotech, Ltd, Guangzhou, China), dehydrated in gradient alcohol and embedded in paraffin (Shanghai Huntz Enterprises Co., Ltd, Shanghai, China). The lobes were then conventionally sliced, dewaxed and stained with hematoxylin and eosin (Guangzhou Wexis Biotech, Ltd) . The pathological states of the liver tissues were observed under an optical microscope (Olympus ix 51; Olympus Corp., Tokyo, Japan) and classified according to the Court of Pathology (Department of Pathology, Guangzhou First People's Hospital, Guangzhou, China). The remaining animals were sacrificed with the same methods on week 12 . Animal care, surgery and sacrifice procedures used in the present study were approved by the Animal Care and Use Committee of Guangzhou First People's Hospital, Guangzhou Medical University (Guangzhou, China).

Pathological diagnostic criteria. The following characteristics were examined: Liver cell degeneration, necrosis, inflammatory cell infiltration, fibrosis, collagen deposition, fibrous septa formation, pseudolobule formation, portal area structure and fibrotic stage, as determined by the well-validated METAVIR scoring system (13). This scoring system assesses fibrotic stage on a five-point scale: F0, no fibrosis; F1, portal fibrosis without septa; F2, portal fibrosis with rare septa; F3, numerous septa without cirrhosis; and F4, cirrhosis.

Statistical analysis. The experimental data were analyzed using the SPSS 13.0 statistical software (SPSS, Inc., Chicago, IL, USA). With numerical variables, the mean value and standard deviation (SD) were calculated. Spearman's rank correlation coefficient was used to assess the correlation between the findings of the histological and elastographic methods.

The diagnostic performances of eSie Touch ${ }^{\mathrm{TM}}$ elasticity imaging and ARFI elastography were assessed using receiver operating characteristic (ROC) curves. These curves were plotted to detect the following: Fibrosis and significant fibrosis ( $F \geq 1$ and 2$)$, severe fibrosis $(F \geq 3)$ and cirrhosis $(F=4)$. Optimal cut-off values were selected to allow the highest sum of sensitivity and specificity. Confidence intervals $(95 \%)$ were calculated for each predictive method. $\mathrm{P}<0.05$ was considered to indicate a statistically significant difference for each procedure.

\section{Results}

Modeling results. A total of 30 cases liver fibrosis were successfully induced and the overall modeling success rate was $86 \%$ (Table I). Three out of the 35 rats succumbed 
Table I. Liver fibrosis modeling results for rats with either 5, 9 or 12 weeks thioacetamide treatment.

\begin{tabular}{lcccccc}
\hline Treatment duration (weeks) & Normal (n) & F1/F2 (n) & F3 (n) & F4 (n) & Mortality (n) & Success rate (\%) \\
\hline 5 & 2 & 8 & 1 & 0 & 1 & 75 \\
9 & 0 & 2 & 7 & 3 & 1 & 92 \\
12 & 0 & 0 & 2 & 7 & 1 & 90 \\
Total & 2 & 10 & 10 & 10 & 3 & $86^{\mathrm{a}}$ \\
\hline
\end{tabular}

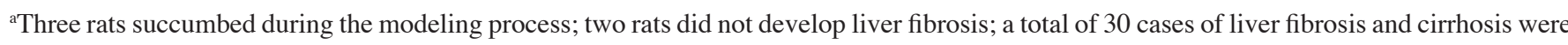
induced; the overall modeling success rate was $86 \%$.

Table II. eSie Touch ${ }^{\mathrm{TM}}$ elasticity imaging data.

\begin{tabular}{lccccr}
\hline & & \multicolumn{3}{c}{ Pathological results } \\
\cline { 3 - 5 } Score & Normal & F1-2 & F3 & F4 & Total \\
\hline 2 & 10 & 0 & 1 & 0 & 11 \\
3 & 0 & 8 & 3 & 0 & 11 \\
4 & 0 & 2 & 6 & 0 & 8 \\
5 & 0 & 0 & 0 & 10 & 10 \\
Total & 10 & 10 & 10 & 10 & 40 \\
\hline
\end{tabular}

during the modeling process and fibrosis was not successfully induced in two cases. All liver specimens in the control group appeared as normal liver tissues. In the modeling group, 10 cases scored F1 or F2, 10 cases scored F3 and 10 cases scored F4.

\section{Pathological results.}

General observation. The liver weights of the control, F1-2, $\mathrm{F} 3$ and $\mathrm{F} 4$ groups were $11.3 \pm 0.48,12.8 \pm 0.79,15.3 \pm 1.57$ and $9.2 \pm 1.55 \mathrm{~g}$, respectively. The difference between groups was tested using one-way analysis of variance, and found to be statistically significant $(\mathrm{P}<0.001)$.

Liver general appearance. The livers of the control group rats were rosy, with smooth surfaces, indurations imperceptible to touch and good elasticity. Examination of the tangent planes revealed a normal red color. The livers of the $\mathrm{F} 1$ and $\mathrm{F} 2$ group rats appeared slightly grey and had a matte-like surface with fine particles, an absence of marked indurations to touch and a marginally greater hardness. Tangent plane analysis revealed an even red color, with fine grainy feeling. The livers of the F3 group rats were dark red, rough and exhibited high hardness. The surfaces were covered with particle protrusions of diameter marginally $<1 \mathrm{~mm}$, with visible small nodules observed on the tangent planes, almost evenly distributed throughout the livers with a dark red color. The livers of the F4 group mice were markedly smaller than those of the other groups. The surfaces were covered with stiff, uneven projections, ranging between $1 \mathrm{~mm}$ and $3 \mathrm{~mm}$, with a lighter color. Indurations and bumps were perceptible by touch. Tangent plane examination revealed scattered distributions of indurations of varied sizes. The tissue color surrounding the indurations was markedly lighter than that of normal liver tissue.
Imaging results. The 10 cases in the control group had an average score of 2 points; group F1/F2 had 3 [80\% (8/10)] and 4 [20\% (2/10)] points; group F3 had 2 [10\% (1/10)], 3 [30\% (3/10)] and 4 [60\% (6/10)] points; and group F4 had 5 points. The eSie Touch ${ }^{\mathrm{TM}}$ elasticity imaging analysis results showed $85 \%$ total accuracy compared with the pathological results as a gold standard (Table II).

VTQ results and pathological standard correlation analysis. The VTQ value difference between the F1 and F2 groups was not statistically significant and the appearances of these two stages were not pathologically distinct. Thus, F1 and F2 were merged as one group. The VTQ values of the 40 rats ranged between 0.69 and 3.56. The mean VTQ values for the control, F1/F2, F3 and F4 groups were $1.08 \pm 0.12,1.51 \pm 0.22$, $1.88 \pm 0.18$ and $2.50 \pm 0.30$, respectively, with statically significant differences between any two groups $(\mathrm{P}<0.05$; Table III).

These results demonstrate that ARFI reliably identified advanced hepatic fibrosis but was insufficient to discriminate between the different stages of minimal and moderate fibroses. Diagnostic accuracy for fibrosis F1 and F2 has been previously demonstrated to be impaired by a marked variation in the shear-wave velocities in these patients (12). Spearman's rank correlation was used to analyze data that were not normally distributed. This revealed a correlation coefficient of $0.969(\mathrm{P}<0.001)$ between the Vs values and the pathological results (Table IV).

eSie Touch $^{\mathrm{TM}}$ elasticity imaging results and pathological standard correlation analysis. Using the eSie Touch ${ }^{\mathrm{TM}}$ semi-quantitative scoring criteria, the 10 cases in the control group all had a score of 2 points; the F1/F2 group had scores of 

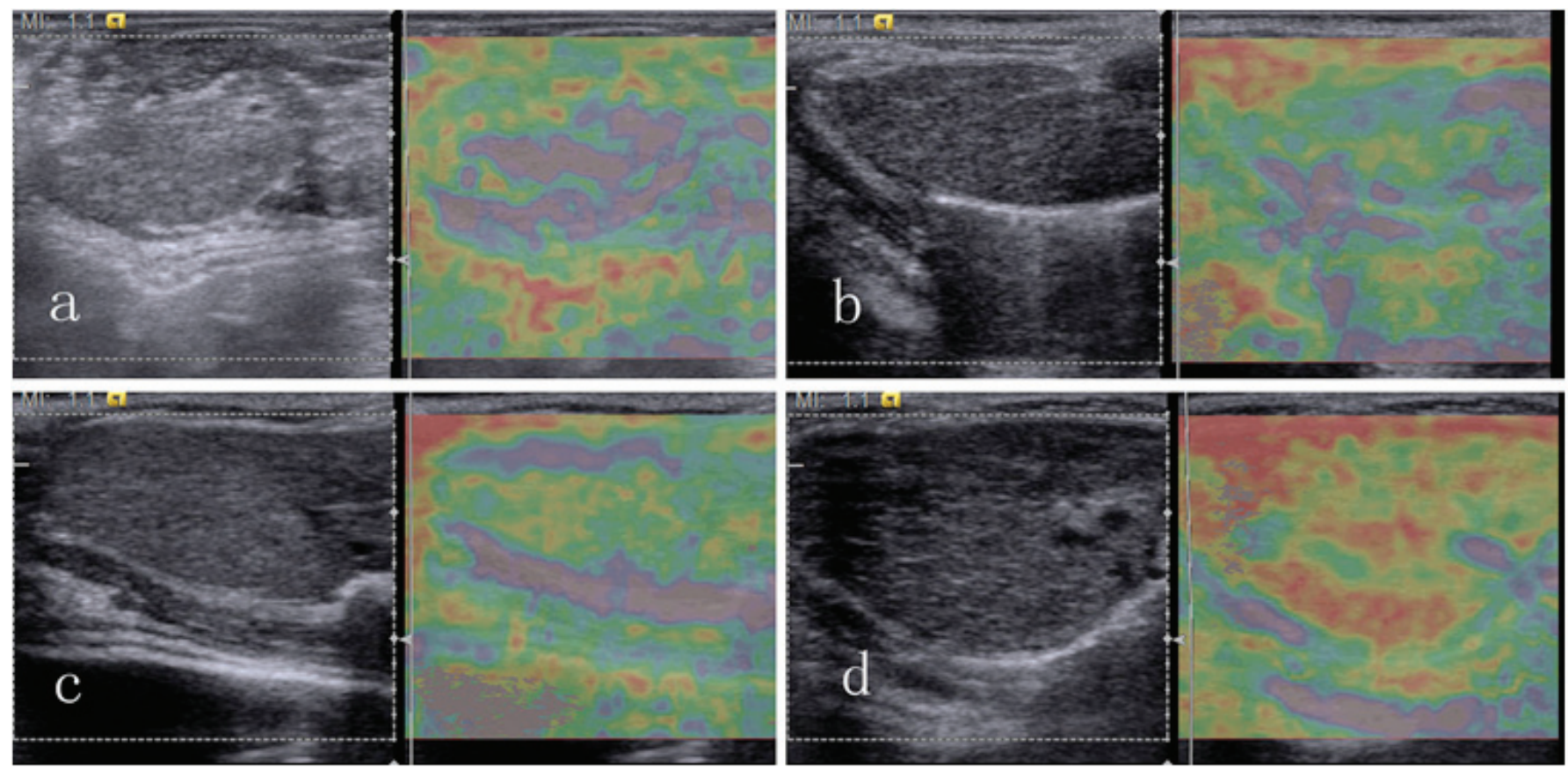

Figure 1. Images indicate the general appearance of lesions for elasticity scores of (a) 2, (b) 3 , (c) 4 and (d) 5 . Lesions with elasticity scores of 1 were not generated in the present study.

3 points $[80 \%(8 / 10)]$ and 4 points $[20 \%(2 / 10)]$; the F3 group had scores of 2 points [10\% (1/10)], 3 points [30\% (3/10)] and 4 points [60\% (6/10)]; and the cases in the F4 group all had a score of 5 points (Table II). The eSie Touch ${ }^{\text {TM }}$ elasticity imaging analysis results had $85 \%$ total accuracy compared with the gold-standard pathological results. Spearman's rank correlation tests demonstrated a correlation coefficient of $0.913(\mathrm{P}<0.001)$ between the elasticity scores and pathological results (Table $\mathrm{V})$.

ROC analysis. ROC analyses were conducted using VTQ and LB staging results as the gold standard, with $\mathrm{F} \geq \mathrm{F} 1 / \mathrm{F} 2, \mathrm{~F} 3$ and F4 as different endpoint packets. The ROC curves were drawn with the VTQ results as quantitative parameters. The maximum total sensitivity and specificity values were used to determine the critical value of the degree of liver fibrosis to analyze the diagnosic efficiency of VTQ (Fig. 2). The results were as follows: $F \geq F 1 / F 2$ : Area under ROC (AUROC) is 1 $(\mathrm{P}<0.001)$ and the best cut-off point is 1.250 (when $\mathrm{Vs}>1.250$, the pathological grading is $\geq F 1 / F 2$ ); $F \geq F 3$ : AUROC is 1 $(\mathrm{P}<0.001)$ and the best cut-off point is 1.685 (when $\mathrm{Vs}>1.685$, the pathological grading is $\geq F 3$ ); and F4: AUROC is 1 $(\mathrm{P}<0.001)$ and the best cut-off point is 2.166 (when Vs $>2.166$, the pathological grading is cirrhosis).

ROC analyses were also conducted using eSie Touch ${ }^{\mathrm{TM}}$ elasticity imaging, with LB staging results as the gold standard (Fig. 3), and the following results were obtained: $F \geq F 1 / F 2$ : AUROC is $0.983(\mathrm{P}<0.001)$ and the best cut-off point is 2.5 (when the elasticity score $>2.5$, the pathological grading is $\geq \mathrm{F} 1 / \mathrm{F} 2) ; \mathrm{F} \geq \mathrm{F} 3$ : AUROC is $0.903(\mathrm{P}<0.001)$ and the best cut-off point is 3.5 (when the elasticity score $>3.5$, the pathological grading is $\geq F 3)$; F4: AUROC is $1(\mathrm{P}<0.001)$ and the best cut-off point is 4.5 (when the elasticity score $>4.5$, the pathological grading is cirrhosis).
Effect contrast between eSie Touch ${ }^{\mathrm{TM}}$ elasticity imaging and $V T Q$. The eSie Touch ${ }^{\mathrm{TM}}$ elasticity imaging and VTQ results were divided into different pathological types according to the previously determined diagnostic boundary values and compared with the gold standard pathological results (Table VI). The accuracy rate of eSie Touch ${ }^{\mathrm{TM}}$ elasticity imaging was $85 \%$, whereas that of VTQ was $100 \%$. The VTQ values of the control, F1/F2, F3 and F4 groups were aggregately distributed and distinguishable from each other (Fig. 4).

\section{Discussion}

Over the past decade, various groups worldwide have made considerable effort to establish reliable and reproducible non-invasive markers of liver fibrosis. In recent years, several studies have assessed the value of ARFI elastography to evaluate liver fibrosis in chronic hepatopathy $(7,14,15)$.

In contrast to LBs, in which analysis is limited to a single core sample, ARFI may be used to noninvasively measure stiffness at multiple points inside the liver. The variation in the excitation location and focal point allows the construction of stiffness images revealing the spatial distribution of shear moduli. Therefore, ARFI has the potential to provide additional information with regard to the spatial distribution of liver stiffness, which may also be diagnostically relevant.

The Siemens ACUSON S2000 with eSie Touch ${ }^{\mathrm{TM}}$ elasticity and VTQ imaging technology was used in the present study. The eSie Touch ${ }^{\mathrm{TM}}$ elasticity imaging technology was established based on ordinary ultrasound diagnostic technology. The low-frequency and low-amplitude vibration wave ultrasonic transducer probe generates an elastic shear wave, the wave velocity of which provides information regarding liver stiffness. Liver tissue fibrosis increases liver hardness 
Table III. Virtual touch tissue quantification data.

\begin{tabular}{lccc}
\hline Group & Minimum & Maximum & Mean $^{\mathrm{a}}$ \\
\hline Control & 0.69 & 1.32 & $1.08 \pm 0.12$ \\
F1/F2 & 0.79 & 2.09 & $1.51 \pm 0.22$ \\
F3 & 1.52 & 2.47 & $1.88 \pm 0.18$ \\
F4 & 1.95 & 3.56 & $2.50 \pm 0.30$
\end{tabular}

${ }^{\text {aThere are statistically significant differences between any two groups }}$ $(\mathrm{P}<0.05)$.

Table IV. Correlation analysis between VTQ and pathological results.

\begin{tabular}{lc}
\hline Pathological result & VTQ Vs value \\
\hline Correlation coefficient & $0.969^{\mathrm{a}}$ \\
Significance (two-tailed) & $<0.001$ \\
$\mathrm{n}$ & 40 \\
\hline
\end{tabular}

Spearman's rank correlation coefficient was used to assess the correlation between the pathological findings and the VTQ results.

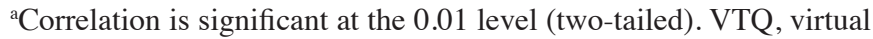
touch tissue quantification; Vs, transverse shear wave velocity.

A

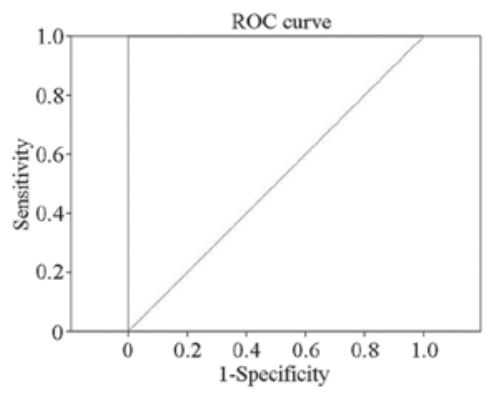

B

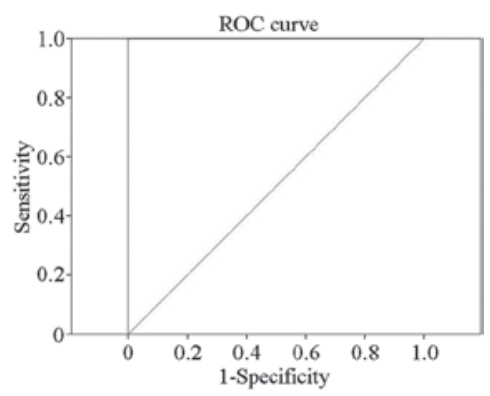

C

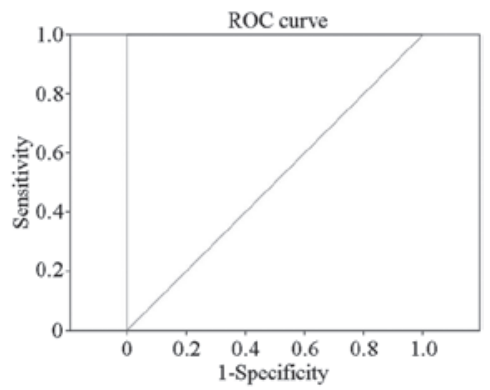

Figure 2. Receiver operating characteristic (ROC) curves for virtual touch tissue quantification results. (A) $\mathrm{F} \geq \mathrm{F} 1-2$; (B) F $\geq \mathrm{F} 3$; (C) F4
A

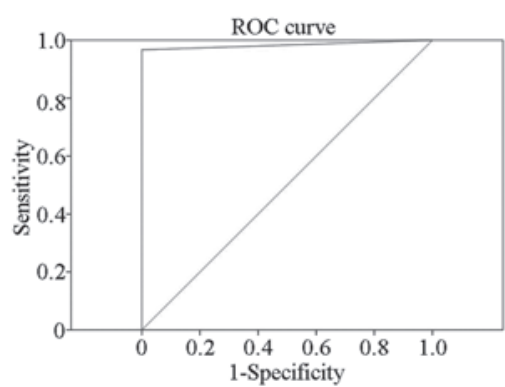

B

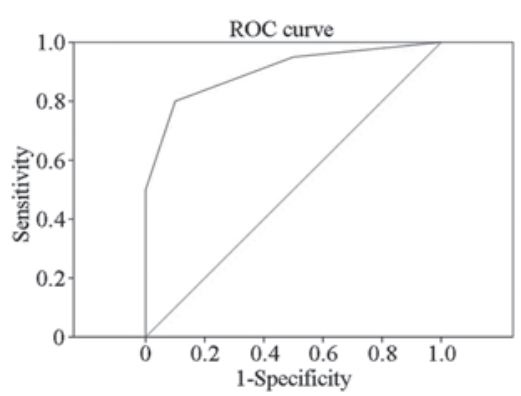

C

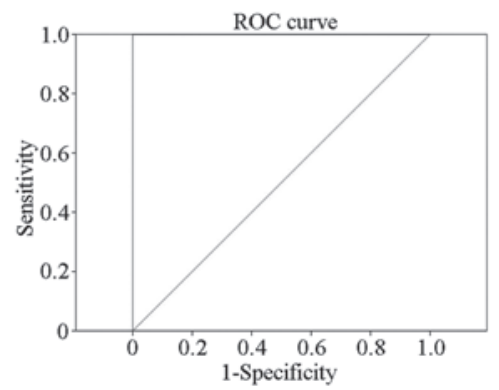

Figure 3. Receiver operating characteristic (ROC) curves for eSie Touch ${ }^{\mathrm{TM}}$ Elasticity Imaging results. (A) F $\geq$ F1-2; (B) F $\geq$ F3; (C) F4.

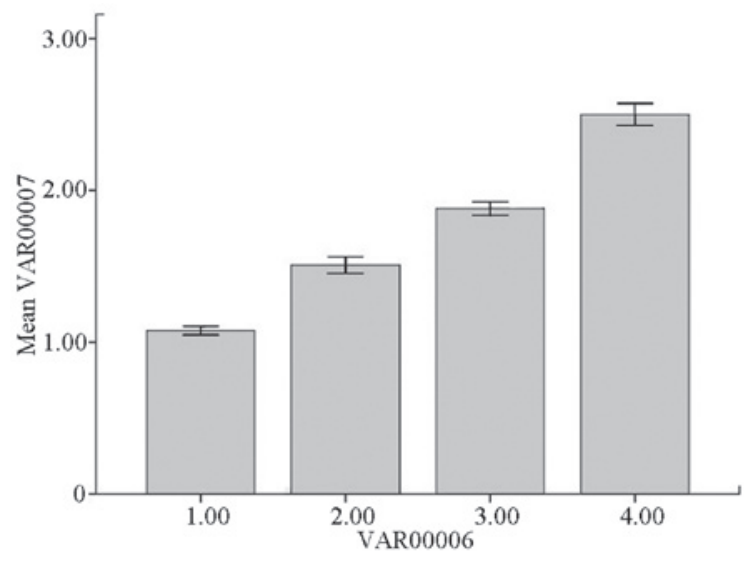

Figure 4. The four groups demonstrated an aggregate distribution.

and changes liver elasticity, which provides the theoretical basis for the possibility of elasticity imaging diagnosis.

eSie Touch ${ }^{\mathrm{TM}}$ elasticity imaging qualitatively reveals stiffness in the ROI, with spatial resolution similar to 2D imaging. The mechanical strain depends on the relative displacement (compressibility) and the stiffness is evaluated by comparing the deformation of the organ between frames. The proprietary filtering and time-processing algorithm technology improves the signal-to-noise and carrier-to-noise ratios, and even small 
Table V. Correlation analysis of elasticity and pathological standard scores.

\begin{tabular}{llcc}
\hline Fibrosis assessment method & \multicolumn{1}{c}{ Parameter } & Elasticity score & Pathology type \\
\hline Elasticity score & Correlation coefficient & 1.000 & $0.913^{\mathrm{a}}$ \\
& significance (two-tailed) & 40.001 & 40 \\
Pathological type & $\mathrm{N}$ & $0.913^{\mathrm{a}}$ & 1.000 \\
& Correlation coefficient & $<0.001$ & 40 \\
\hline
\end{tabular}

Spearman's rank correlation coefficient was used to assess the correlation between the pathological findings and the elastographic methods

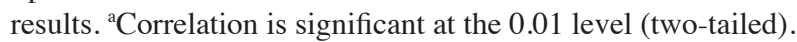

Table VI. Results of eSie Touch ${ }^{\mathrm{TM}}$ elasticity imaging and VTQ.

\begin{tabular}{|c|c|c|c|c|c|c|c|c|}
\hline \multirow{2}{*}{$\begin{array}{l}\text { Pathological } \\
\text { results } \\
\text { (Gold standard) }\end{array}$} & \multicolumn{4}{|c|}{ eSie Touch ${ }^{\mathrm{TM}}$ elasticity imaging prediction } & \multicolumn{4}{|c|}{ VTQ prediction } \\
\hline & Normal & F1-2 & F3 & F4 & Normal & F1-2 & F3 & F4 \\
\hline Normal (n) & 10 & 0 & 0 & 0 & 10 & 0 & 0 & 0 \\
\hline$(\%)$ & 25.00 & 0.00 & 0.00 & 0.00 & 25.00 & 0.00 & 0.00 & 0.00 \\
\hline $\mathrm{F} 1-2(\mathrm{n})$ & 0 & 8 & 2 & 0 & 0 & 10 & 0 & 0 \\
\hline (\%) & 0.00 & 20.00 & 5.00 & 0.00 & 0.00 & 25.00 & 0.00 & 0.00 \\
\hline $\mathrm{F} 3(\mathrm{n})$ & 1 & 3 & 6 & 0 & 0 & 0 & 10 & 0 \\
\hline$(\%)$ & 2.50 & 7.50 & 15.00 & 0.00 & 0.00 & 0.00 & 25.00 & 0.00 \\
\hline $\mathrm{F} 4(\mathrm{n})$ & 0 & 0 & 0 & 10 & 0 & 0 & 0 & 10 \\
\hline$(\%)$ & 0.00 & 0.00 & 0.00 & 25.00 & 0.00 & 0.00 & 0.00 & 25.00 \\
\hline
\end{tabular}

VTQ, virtual touch tissue quantification.

displacements (deformations) may be detected. The operator does not need to pressure the probe since the proprietary imaging technology detects the compressed tissue deformation caused by breathing and heart beating, in addition to providing a visually qualitative method and enhancing the patient's comfort. Real-time QF is used to evaluate the accuracy of displacement. A higher QF indicates a small illusion of the entire displacement, whereas a lower QF indicates a reduced diagnostic value by the illusion. The rapid respiratory movement of rats reduces the QF; thus, monitoring the breathing motion of the rats and achieving probe stability during examination of the rat's liver is required.

The VTQ technique is determined by ARFI principles. This novel technology is used to detect and evaluate tissue stiffness. The principle is to produce an acoustic shear wave in the biological tissue using viscoelastic focused ultrasonic beam modulation, where the signal of the shear wave in the tissue is recorded by a special electronic system. The rapid decay of the radiation force in the focused area results in the limitation of the shear wave to small regional areas. Therefore, the machine obtains the propagation velocity of the shear wave at low frequency in the ROI. The tissue elasticity modulus is estimated through the detected shear wave propagation. The system uses a standard ultrasonic probe and a depth-adjustable sampling frame with a length of $\sim 5 \mathrm{~mm}$. The probe sends short-duration acoustic pulses to generate localised, micron-scale displacements in the organ. The displacement in the ROI is tracked using the ultrasonic probe. The results are expressed by the shear-wave velocity $(\mathrm{m} / \mathrm{sec})$, which indicates the stiffness of the tissue (16).

The present study demonstrated a significant positive correlation between ARFI elastographic velocity and liver fibrosis severity in rats, with the VTQ values of the control, F1/F2, F3 and F4 groups aggregately distributed and distinct from each other. Fibrogenesis is assumed to be heterogeneous in the liver as fibrosis variation in different hepatic regions is a known phenomenon in LB samples $(17,18)$ and the distribution curves of the control, F1/F2, F3 and F4 groups overlap. Despite the small overlapping range, the normal, F1/F2, F3 and liver cirrhosis stages may be distinguished. The present study suggests that a rat with mean VTQ value $<1.250 \mathrm{~m} / \mathrm{sec}$ (the best VTQ value cut-off point distinguishing normal liver tissue and fibrotic liver tissue) and an associated eSie Touch ${ }^{\mathrm{TM}}$ score of 2 may be excluded from liver fibrosis diagnosis. If the mean VTQ value of a rat is between 1.250 and $1.685 \mathrm{~m} / \mathrm{sec}$ with a score of 3 on the eSie Touch ${ }^{\mathrm{TM}}, \mathrm{F} 1 / \mathrm{F} 2$ liver fibrosis may be diagnosed. If ARFI values are between 1.685 and $2.166 \mathrm{~m} / \mathrm{sec}$, with a score of 4 on the eSie Touch ${ }^{\mathrm{TM}}$, F3 liver fibrosis may be diagnosed. If the VTQ value is $>2.166 \mathrm{~m} / \mathrm{sec}$ with a score of 5 on the eSie Touch ${ }^{\mathrm{TM}}$, the diagnosis is liver fibrosis grade F4. 
This technique meets the requirements for the early diagnosis of liver fibrosis.

Statistical analysis revealed that the accuracy rates of eSie Touch $^{\text {TM }}$ elasticity imaging and VTQ were 85 and 100\%, respectively. Thus, VTQ is superior to eSie Touch ${ }^{\mathrm{TM}}$ elasticity imaging in terms of grading liver fibrosis.

The VTQ data in the present study are similar to those observed in comparative studies of patients with liver fibrosis and healthy controls. In other studies, the mean \pm SD vs. the values for the controls were found to be $1.08 \pm 0.13$ (19), $1.15 \pm 0.21$ (20) and $1.08 \pm 0.15 \mathrm{~m} / \mathrm{sec}$ (21), with values for healthy volunteers similar to those of control rats in the present study $(1.08 \pm 0.12)$. The cut-off values used in the present study to identify the controls and classify the fibrosis stage (F0 versus $\mathrm{F} 1-\mathrm{F} 4,1.250 \mathrm{~m} / \mathrm{sec}$; F0-F2 versus F3/F4, $1.685 \mathrm{~m} / \mathrm{sec}$; and $\mathrm{F} 0-\mathrm{F} 3$ versus $\mathrm{F} 4,2.166 \mathrm{~m} / \mathrm{sec}$ ) are close to those determined by Karlas et al (12), who calculated the cut-off values for patients with fibrosis as follows: F0 versus F1-F4, $1.40 \mathrm{~m} / \mathrm{sec}$; F0/F1 versus F2-F4, $1.70 \mathrm{~m} / \mathrm{sec}$; F0-F2 versus F3/F4, $1.70 \mathrm{~m} / \mathrm{sec}$; F0-F3 versus F4, $2.13 \mathrm{~m} / \mathrm{sec}$.

Therefore, the eSie Touch ${ }^{\mathrm{TM}}$ elasticity imaging and VTQ techniques may be successfully adopted to assess the extent of liver stiffness and the two techniques are expected to replace LB. VTQ technology provides multiple advantages, including non-invasiveness, rapid process, low cost and dynamic evaluation. The technique is advantageous in examining liver fibrosis, particularly for patients who are obese or have a narrow intercostal space, rendering examination of fibrosis by TE difficult. The present study is important for clinical research, as further studies based on the present study may be conducted addressing the clinical aspect of fibrosis.

\section{Acknowledgements}

This study was supported by the Guangdong Natural Science Foundation (no. 9151008901000201), the Guangdong Social Development Project (no. 2011 NO.02), the Guangdong Science and Technology Project (no. 01557050170800038), and the Guangzhou Medical Science and Technology Project (nos. 20121A011027, 20131A010004 and 20131A011014).

\section{References}

1. Friedman SL: Liver fibrosis - from bench to bedside. J Hepatol 38 (Suppl 1): S38-S53, 2003.

2. World Health Organization: Hepatitis B. World Health Organization Fact Sheet 204 (Revised August 2008). http://www. who.int/mediacentre/factsheets/fs204/en/index.html. Accessed February 25, 2012.
3. Goldstein ST, Zhou F, Hadler SC, Bell BP, Mast EE and Margolis HS: A mathematical model to estimate global hepatitis B disease burden and vaccination impact. Int J Epidemiol 34: 1329-1339, 2005.

4. Bonis PA, Friedman SL and Kaplan MM: Is liver fibrosis reversible? N Engl J Med 344: 452-454, 2001.

5. Bravo AA, Sheth SG and Chopra S: Liver biopsy. N Engl J Med 344: 495-500, 2001.

6. Rockey DC, Caldwell SH, Goodman ZD, Nelson RC and Smith AD; American Association for the Study of Liver Diseases: Liver biopsy. Hepatology 49: 1017-1044, 2009.

7. Friedrich-Rust M, Wunder K, Kriener S, et al: Liver fibrosis in viral hepatitis: noninvasive assessment with acoustic radiation force impulse imaging versus transient elastography. Radiology 252: 595-604, 2009.

8. Zhai L, Palmeri ML, Bouchard RR, Nightingale RW and Nightingale KR: An integrated indenter-ARFI imaging system for tissue stiffness quantification. Ultrason Imaging 30: 95-111, 2008.

9. Sandrin L, Fourquet B, Hasquenoph JM, et al: Transient elastography: a new noninvasive method for assessment of hepatic fibrosis. Ultrasound Med Biol 29: 1705-1713, 2003.

10. Yang L, Ma F, Liu YY and Dang YY: Value of ultrasonic elastography in qualitative diagnosis for breast parenchymatous tumors. J Tongji Univ (Med Sci) 2008: 135-137, 2008.

11. Itoh $\mathrm{A}, \mathrm{Ueno} \mathrm{E}$, Tohno $\mathrm{E}$, et al: Breast disease: clinical application of US elastography for diagnosis. Radiology 239: 341-350, 2006.

12. Karlas T, Pfrepper C, Wiegand J, et al: Acoustic radiation force impulse imaging (ARFI) for non-invasive detection of liver fibrosis: examination standards and evaluation of interlobe differences in healthy subjects and chronic liver disease. Scand J Gastroenterol 46: 1458-1467, 2011.

13. Bedossa P and Poynard T: An algorithm for the grading of activity in chronic hepatitis C. The METAVIR Cooperative Study Group. Hepatology 24: 289-293, 1996.

14. Sporea I, Sirli RL, Deleanu A, et al: Acoustic radiation force impulse elastography as compared to transient elastography and liver biopsy in patients with chronic hepatopathies. Ultraschall Med 32 (Suppl 1): S46-S52, 2011.

15. Rizzo L, Calvaruso V, Cacopardo B, et al: Comparison of transient elastography and acoustic radiation force impulse for non-invasive staging of liver fibrosis in patients with chronic hepatitis C. Am J Gastroenterol 106: 2112-2120, 2011.

16. Palmeri ML, Sharma AC, Bouchard RR, Nightingale RW and Nightingale KR: A Finite-Element Method Model of Soft Tissue Response to Impulsive Acoustic Radiation Force. IEEE Trans Ultrason Ferroelectr Freq Control 52: 1699-1712, 2005.

17. Goldstein NS, Hastah F, Galan MV and Gordon SC: Fibrosis heterogeneity in nonalcoholic steatohepatitis and hepatitis $\mathrm{C}$ virus needle core biopsy specimens. Am J Clin Pathol 123: 382-387, 2005.

18. Mehta SH, Lau B, Afdhal NH and Thomas DL: Exceeding the limits of liver histology markers. J Hepatol 50: 36-41, 2009.

19. Takahashi H, Ono N, Eguchi Y, et al: Evaluation of acoustic radiation force impulse elastography for fibrosis staging of chronic liver disease: a pilot study. Liver Int 30: 538-545, 2010.

20. Popescu A, Sporea I, Sirli R, et al: The mean values of liver stiffness assessed by Acoustic Radiation Force Impulse elastography in normal subjects. Med Ultrason 13: 33-37, 2011.

21. Horster S, Mandel P, Zachoval R and Clevert DA: Comparing acoustic radiation force impulse imaging to transient elastography to assess liver stiffness in healthy volunteers with and without valsalva manoeuvre. Clin Hemorheol Microcirc 46: 159-168, 2010. 